\title{
ALGUNAS CONSIDERACIONES SOBRE LA EXPRESIÓN "DISCURSO LITERARIO"
}

El uso de la expresión "discurso literario" para designar los textos que se reconocen y se utilizan como literatura comporta una doble dificultad: por un lado, la definición de discurso, y por otro, el problema de su tipología, es decir, la atribución del carácter de literario a uno de los tipos de discurso.

Gencralmente, cuando se habla de discurso sc lo hace equivalente a una unidad lingüística de dimensiones mayores que las de la oración; por esta razón, los analistas han tenido que producir ias lingüísticas del texto o del discurso, las cuales han tratado de sobrepasar los límites impuestos por la lingüística de la lengua. Las lingüísticas del tex to o del discurso tratan de rebasar el nivel de análisis de Ja oración -que es el nivel superior en la lingüística estructural-. y constituyen un esfuerzo por cscapar a la doble reducción del lenguajc: a la lengua y al código; constituyen también una "tentativa para reintroducir el sujeto y la situación de comunicación excluidos en virtud del postulado de la inmanencia" (Maldidier et al.,1972: 118).

Las lingüísticas del discurso, desde la de Harris hasta las estudiadas por van Dijk, siguen considerando el discurso fundamentalmente como compuesto por signos lingüísticos. Es indudable que los discursos están formados por signos - dice Foucault- "pero lo que hacen es más que utilizar esos signos para indicar cosas. Es ese más lo que los vuelve irreductibles a la lengua y al habla. Es ese más lo que hay que revelar y hay que describir" (1970:81). El estudio del discurso, por otro lado, de ninguna forma puede hacerse equivalente a la lingüística del habla, pues el habla, en el sentido de Saussure, es la manifestación lingüística inmediata de una intención de comunicación que depende de la decisión subjetiva del hablante, mientras que el discurso es otra cosa: el concepto de discurso 
se opone al de habla porque posee una estabilización pública y normativa, y un estatuto institucional (Stierle 1977: 425). Examinemos esas características.

Partamos de una primera definición: el discurso es un conjunto de enunciados. Un enunciado no es lo mismo que una frase o una proposición, pues éstas son las unidades que la gramática y la lógica reconocen en un conjunto de signos, y que pueden caracterizarse por sus elementos o por las reglas de construcción que los unen. Enunciado, por su parte, es:

la modalidad de existencia propia de este conjunto de signos: modalidad que le permite ser algo más que una serie de trazos, algo más que una sucesión de marcas sobre una sustancia, algo más que un objeto cualquiera fabricado por un ser humano; modalidad que le permite estar en relación con un dominio de objetos, prescribir una posición definida a todo sujeto posible, estar situado entre otras actuaciones verbales, estar dotado en fin de una materialidad repetible (Foucault 1970: 180).

Si se habla de discurso político, discurso filosófico, discurso literario, etc., debe entonces existir alguna forma de agrupar los discursos bajo esos grandes títulos. Cómo fundar la unidad de los enunciados: ésa es la pregunta que está detrás de toda clasificación, y a ella trata Foucault de responder en La arqueología del saber por medio de ciertas hipótesis que él mismo contradice inmediatamente. La primera hipótesis es que "los enunciados diferentes en su forma, dispersos en el tiempo, constituyen un conjunto si se refieren a un solo y mismo objeto"; pero en lugar de encontrar la permanencia y la singularidad de un objeto, lo que hay es una dispersión de objetos. La segunda sostiene que lo que define la unidad es cierto tipo de enunciación; pero al analizar un tipo de discurso se encuentran formulaciones diferentes y funciones heterogéneas, de mancra que no puede formarse una figura única que asimile a través del tiempo un texto ininterrumpido. La tercera dice que los grupos pueden establecerse determinando el sistema de conceptos que se encuentran en los enunciados; pero tal vez la unidad no esté del lado de la coherencia de los conceptos sino por "su emergencia simultánea o sucesiva, de desviación, de la distancia que los separa y eventualmente 
de su incompatibilidad"; es decir, estamos siempre ante conceptos que difieren tanto por su estructura como por sus reglas de combinación, que se ignoran o se excluyen unos a otros. Por último, la hipótesis que plantea la unidad de los enunciados en función de la identidad y persistencia de los temas; pero, lo que se encuentra en el análisis es más bien las posibilidades que permiten la activación de temas incompatibles, o la incorporación de un mismo tema o conjuntos distintos (Foucault 1970: 52-62). De aquí que, para encontrar la regularidad tengan que describirse estas dispersiones. Si puede describirse tal sistema de dispersión, si puede además definirse una regularidad entre los objetos, los tipos de cnunciación, los conceptos, las elecciones temáticas, entonces estamos en prescncia de una formación discursiva.

Con el concepto de formación discursiva, la definición de discurso se completa; discurso es:

el conjunto de enunciados en tanto que dependan de la misma formación discursiva; no forma una unidad retórica o formal, y cuya aparición o utilización en la historia podría señalarse (y explicarse llegado el caso); está constituido por un número limitado de enunciados para los cuales pucde definirse un conjunto de condiciones de existencia (Foucault 1970: 198).

Un discurso se caracteriza, según este mismo autor, por la regularidad de una práctica, una práctica compleja y diferenciada que obedece a reglas y transformaciones analizables. Este concepto de práctica discursiva añade una precisión, pues se opone al discurso en su concepción ordinaria, como conjunto de clementos significantes que remiten o a contenidos o a representaciones; el concepto de práctica discursiva se define como "un conjunto de reglas anónimas, históricas, siempre determinadas en el tiempo y el espacio, que han definido en una época dada, y para un área social, geográfica o lingüística dada, las condiciones de ejercicio de la función enunciativa" (Ibid).

Un discurso, entonces, se caracteriza no por tratar acerca de determinados objetos, sino por la mancra en que forma sus objetos; una formación discursiva tiene su origen en un conjunto de relaciones establecidas entre instancias de emer- 
gencia, de delimitación y de especificación. Los objetos del discurso no existen naturalmente sino que surgen en condiciones históricas precisas: un objeto de discurso surge cuando varias personas pueden decir de él cosas distintas, cuando se inscribe en un dominio de parentesco con otros objetos. El objeto existe en las condiciones de un haz complejo de relaciones, las cuales se establecen "entre instituciones, procesos económicos y sociales, formas de comportamiento, sistemas de normas, técnicas, tipos de clasificación, modos de caracterización" (Ibid: 74 ).

Con estas consideraciones, Foucault escapa al peligro del análisis puramente interno, tal como lo realiza el estructuralismo, cuya ley de funcionamiento es intradiscursiva; en lugar de eso, sale del discurso para dar cuenta del discurso.

Así, si se habla de discurso literario, o más bien, del conjunto de discursos que designamos como literarios, esta designación no obedece al hecho de que traten todos del mismo objeto, ni a que sean resultado de un tipo particular de erunciación, ni a que todos esos discursos utilicen el mismo diccionario de nociones y conceptos, ni, finalmente, porque comparten las temáticas; si hablamos de discursos literarios es porque todos esos discursos pertenecen a la formación discursiva que llamamos "literatura", o, dicho en las palabras de Stierle, "por la relación que mantienen con un esquema discursivo preexistente, que se extiende más allá del discurso individual y concreto y que es capaz, como esquema del mundo simbólico de los actos, de orientar tanto su producción como su recepción" (Stierle 1977: 426).

El hecho de que no exista una característica puramente textual para calificar un discurso como literario, ha llevado a algunos investigadores a plantear un problema de mucho interés. Jens lhwe, por ejemplo, declara que los objetos considerados como textos literarios, es décir, lo que es literatura, no puede determinarse sobre la base de alguna propiedad inherente, sino que su pertenencia al conjunto de los discursos literarios depende de las normas socio-culturales aceptadas en una sociedad particular. Añade:

la expresión "literatua" usualmente aparece en contextos en los que las personas actúan bajó la presión de instancias culturales, 
instituciones de la sociedad ... En esas circunstancias, es obvio que algo como un "status literario" de una expresión verbal no existe (Ihwe 1973: 313).

Todorov, por su parte, señala que cuando uno se pregunta de dónde viene la certidumbre de que existe una entidad llamada literatura, la respuesta es que viene de la experiencia, del aprendizaje en la escuela; sabemos que una entidad "literatura" funciona en el nivel de las relaciones sociales, pero:

¿qué se prueba con eso? Que en un sistema más amplio, en la sociedad o en la cultura, existe un elemento identificable al cual nos referimos con la palabra "literatura". ¿Demostramos con eso que todos los productos particulares que asumen esa función participan de una naturaleza común, que tenemos igualmente el derecho a identificar? De ningún modo (1975: 353).

No es obvio, dice Todorov, que la literatura pueda identificarse por sus características lingüísticas, pues, cn ese nivel, el discurso literario comparte rasgos con otros discursos no literarios; más bien, "Io que une los diferentes géneros literarios en una 'literatura' (. . .) es su uso, su función en la vida social. La literatura es una entidad sociológica c históricamente determinada" (Todorov 1979:510).

Más adelante me referiré al tema de lo institucional en literatura, problema muy ligado con lo dicho por Ihwe y por Todorov; por ahora, lo que importa de su posición es que refuerza la idea de la ausencia de unidad interna en los tipos de discurso $y^{y}$ la idea de una dispersión, tanto en lo que sc refiere a objetos, como a enunciaciones, alfabeto de nociones y temáticas.

Desde un ángulo distinto, podemos considerar los textos literarios como procesos significantes, es decir, como procesos que tienen como base la facultad de lenguajc, la capacidad de establecer relaciones de significación, de representar lo real por signos y de comprender esos signos como representantes de lo real. Como señala Verón, la producción social de la significación consiste en una red que es, para todos los efectos prácticos, infinita; por lo tanto, todo discurso, literario o no, es lugar de manifestación de las determinaciones sociales; no. es un objeto homogéneo sino plural, susceptible de una multi- 
plicidad de lecturas por ser el punto de intersección de varios sistemas diferentes y heterogéneos.

Los procesos significantes, por lo tanto, no están determinados unívocamente, sino que al ser la intersección de prácticas diversas, son objetos heterogéneos susceptibles de producir significaciones también diversas. Los discursos lingüísticos (o procesos significantes manifestados por medio de la materia lingüística), al estar también multívocamente determinados, no pueden analizarse con la única consideración de que son series de frases o de oraciones que remiten al código de la lengua. Contrariamente a lo que señala Saussure, los discursos no tienen una sola dimensión, no son lineales; son más bien, como indica Verón, "paquetes significantes complejos que recorren las redes sociales de la significación".

En resumen, existe, por un lado, la posibilidad de múltiples lecturas para un texto dado, especialmente si se trata de un texto reconocido como literario; por otro lado, existe la normatividad impuesta por la formación discursiva en la cual ese texto está inserto, la cual privilegia una lectura y reprime las demás. Estas dos posiciones pueden parecer contradictorias, pero en realidad no es así, puesto que los textos, como lugares de lucha, como "síntesis de múltiples determinaciones", no pueden reducirse simplemente a la manifestación de los esquemas discursivos que les subyacen; su potencialidad de significación permite la emergencia de sentidos en otros planos, de lecturas en otras direcciones en las cuales las significaciones se ramifican, establecen contactos y actualizan virtualidades; "precisamente porque el discurso no puede reducirse nunca sin ruptura a la manifestación de un esquema discursivo, es, bajo su aspecto concreto, siempre y al mismo tiempo un no discurso" (Stierle 1977: 427).

Es también Foucault quien ha mostrado el conflicto entre las posibilidades infinitas de un mundo anárquico de las significaciones, en el que todas las combinaciones son posibles, y la sujeción de los textos al orden del discurso. En su lección inaugural en el College de France, Foucault muestra que el conflicto se resuelve generalmente a favor del orden, ya que

en toda sociedad la producción del discurso está a la vez controlada, seleccionada y redistribuida por un cierto número de procedi- 
mientos que tienen por función conjurar los poderes y peligros, dominar el acontecimiento aleatorio y esquivar su pesada y temible materialidad (1973: 11).

Menciona el autor tres grupos de procedimientos de control de los discursos; el primero de ellos está formado por los procedimientos que sc cjercen desde el exterior del discurso y consta de los siguientes:

-lo prohibido: "se sabe que no se tiene derecho a decirlo todo, que no se puede hablar de todo en cualquier circunstancia, que' cualquiera, en fin, no puede hablar de cualquier cosa" (Ibid). Los puntos en los que este procedimiento tradicionalmente se ha concentrado son la sexualidad y la política, más generalmente, en el deseo y el poder. Como ilustración de este procedimiento de control en el campo de lo literario pueden citarse los casos de Bataille, siempre excluido de los textos y manuales de literatura, escritor maldito cuyo nombre apareció en sus novelas Fistoria del ojo y Madame Edwarda sólo hasta después de su muerte, y el de José Revueltas, quien no puede quitarse todavía la caracterización de "mal escritor";

-el establecimiento de una línca de separación entre razón y locura: "el loco es aquel cuyo discurso no puede circular como el de los otros". Este procedimiento, al igual que el anterior, ha perdido su vigencia, pero nombres como el de Artaud o el de Jorge Cuesta nos muestran que ha sido muy elicaz. Accrca del primero, Durozoi señala que siempre la crítica nos ha prohibido el acceso a los textos de Artaud, textos singulares, que rompen por completo con lo que habitualmente llamamos "literatura". Artaud, igual que Bataille, es otro cscritor maldito; pero son esa misma clasificación, lo que se hace es inscribirlos en marcos rígidos y asegurar su recuperación por el sistema cultural dominante. Si Artaud es un loco, su discurso es peligroso; por lo tanto, debe silenciarse, cerrársele la boca, así como se le "cerró la boca a Baudelaire, a Edgar Poe, a Gérard de Nerval, y al conde de Lautréanont. Porque se tenía miedo de quc su poesía saliera de los libros $y$ trastornara la realidad" (Artaud);

- establecimiento de una separación entre lo verdadero y lo falso: primacía de lo verosímil. Así como los otros pro- 
cedimientos de exclusión, la voluntad de verdad tiene también un soporte institucional: las prácticas escolares, las editoriales, las bibliotecas; pero también "por la forma que tiene el saber de ponerse en práctica en una sociedad, en la que es valorizado, distribuido, repartido y en cierta forma atribuido" (Foucault 1973: 18). Toda literatura occidental, añade, desde hace siglos ha buscado apoyo sobre lo natural, sobre lo verosímil, sobre la sinceridad, y también sobre la ciencia. Recuérdense los puntos de apoyo que menciona Barthes, encontrados en Sarrasine, de Balzac: en la historia, la geografía, la historia del arte, etc.

Un segundo grupo de procedimientos de control, procedimientos internos al discurso, está formado por los siguientes:

-el comentario de textos: el comentario permite construir nuevos discursos, pero no tiene otra finalidad que decir lo que ya estaba dicho; es decir, el comentario de textos permite decir otra cosa acerca del texto pero con la condición de que sea el texto mismo el que se repita. Este procedimiento "conjura el azar del discurso (...) La multiplicidad abierta, el azar, son transferidos desprovistos de aquello de que habría peligro si se dijese" (Ibid: 24). Además, no cualquier persona puede comentar un texto, pues no cualquiera puede encontrar el "verdadero" significado de una obra: sin embargo, como el comentario es un ejercicio universitario, para controlar su producción, para conjurar el azar, para eso está el manual;

-el principio del autor: el autor se considera como un "principio de agrupación del discurso, como unidad y origen de sus significaciones, como foco de su coherencia" (Ibid.). Al autor se le pide que dé cuenta de la unidad del texto, que revele su sentido oculto; por ello se recurre al estudio de su vida personal, de su historia;

- la disciplina. Estos dos principios, el del comentario y el del autor, se encuentran con un tercero: el de la disciplina. La disciplina fija sus límites al discurso por medio del juego de una identidad "que tiene la forma de una reactualización permanente de las reglas" (Ibid: 31). Existe, pues, una disciplina que estudia los textos literarios (que adopta en las universidades diversos nombres, entre ellos el de teoría litera- 
ria); csta aisciplina, junto con todo el soporte formado por los profesores de literatura, estudiantes, suplementos culturales, revistas, antologías, historias de la literatura, editoriales, críticos, etc., configura el sistema de reglas que detcrmina cuáles textos son literarios y quićn puede hablar sobre ellos. Este sistema de reglas, que produce el conjunto de discursos sobre la literatura, es lo que llamaremos la institución de la literatura.

El último grupo de procedimientos de control de que habla Foucault no consta de procedimientos encaminados a conjurar los poderes que conllevan, ni a controlar sus apariciones; su función es imponer a los individuos que producen los discursos ciertas reglas; con ello aseguran cl acceso a la litcratura, en el caso de los discursos literarios, sólo a determinados sujetos: "nadie entrará en el orden del discurso si no satisface ciertas exigencias o si no está, de cntrada, calificado para hacerlo" (Ibid: 32). Como en cualquier otra institución pero de una forma más manifiesta, en la literatura ese sistema de rcglas se encuentra encarnado en ciertos individuos, que desempeñan el papel de pontífices. Octavio Paz es uno de ellos para las letras hispánicas contemporáneas; como ilustración puede mencionarse su papel en la valoración como poeta de José Carlos Becerra: dicc en Pocsía en movimiento: "Hasta hace unos días no sabía nada de Becerra. Acabo de leer unos clantos poemas suyos y su fucgo templado me hizo pensar en un poeta joven y ya maduro." En el prólogo a la obra completã de Bccerra, dice cl mismo Pay: "Pecerra fuc uno de los jóvenes poetas incluidos en Poesía en movimiento. Fue el primer reconocimicnto público de su obra, algo así como la declaración de su mayoría de edad poética. Las antologías son el equivalente modcrno de las ordalías y las otras pruebas de los antiguos ritos de pasaje." Si las antologías son equivalentes a las ordalías, los antologistas son los jueces; así, los verdaderos poetas jóvenes de México, los reconocidos como poetas, los autorizados para producir poesía, son los que Zaid admitió en su Asamblea de poetas.

Como ya se ha señalado antes, las antologías, los editores, los críticos, los suplementos culturales, los profesores, los investigadores literarios, el sistema de premios, las escuelas 
de letras, los congresos, etc., todo ello conforme la institución de la literatura. El uso del concepto de institución es muy extenso en sociología; allí se denomina institución un conjunto de normas que se aplican a un dominio particular de actividades y definen una lcgitimidad que se expresa en un cierto código. Este concepto, tomado en esa acepción no nos proporciona los instrumentos pará aprehender una situación histórica concreta, pues omite considerar lo que forma la base matcrial de cualquier institución; omite también los efectos que puede producir en la reproducción de las relaciones sociales y la función que cumple en el interior de esas relaciones (Dubois 1978: 32 y ss).

La intervención de las instituciones y la eficacia de esa intervención no pueden apreciarse en bloque, puesto que existen varios niveles; bajo su forma más visible, aparecen funcionalmente como modos de organización que aseguran la permanencia de los individuos en una colectividad dada y los integran al sistema de producción. Cada institución cubre un sector específico de actividades y de prácticas; sin embargo, ejercen un control desigual sobre esas actividades y prácticas. La eficacia de cada institución se logra por la imposición de sistemas de normas y valores; y es aquí donde las instituciones aparecen como lugares de dominación y de subordinación ideológica, lo cual no es resultado sólo de los discursos que difunden y que producen, sino que "el carácter de imposición de las instituciones está ya inscrito en su modo de recorte de la realidad de las prácticas sociales, en la manera en que fijan, sobre el terreno de una legitimidad, las condiciones de posibilidad y de ejercicio de esas prácticas" (Ibid:33).

Como un ejemplo de institución, Evrard habla de la psiquiatría, y con ello no se refiere solamente a lo que la psiquiatría pretende ser, o la suma de conocimientos de los psiquiatras, o a las prácticas psiquiátricas reales. Se refiere también a las representaciones que nos hacemos, tanto nosotros, desde afuera, como los psiquiatras y sus pacientes. Es decir, de la institución también forman parte las representaciones imaginarias, los mitos, los prejuicios, todos los aspectos subjetivos e irracionales que la ciencia rechaza. Para Bernard, una institución, en el sentido amplio, es todo sitio 
donde se opere la reproducción de las relaciones sociales dominantes; cada institución es una red simbólica, "socialmerite sancionada, donde se combinan en proporciones y relaciones variables un componente funcional y un componente imaginario" (Bernard 1976: 36).

El carácter de institución siempre ha sido rechazado en todo tratamiento de la litcratura; los discursos sobre lo literario no sólo rechazan ese carácter, sino que generalmente presentan su objeto como una esencia, sin contactos con la base histórica y social. Lo que añadiría cl análisis de lo litcrario como institución sería "descubrir que no existe la Litcratura, sino prácticas cspeciales, que operan a la vez sobre el lenguaje y sobre lo imaginario y cuya unidad no se realiza más que a ciertos niveles de funcionamiento y de inserción en la estructura social" (Dubois 1978: 11).

El momento fundador de toda institución es cl momento de aparición de una legitimidad que sc elabora en forma interna y que designa estas actividades como autónomas y distintivas. En el campo de lo literario, csa legitimidad se clabora internamente a la esfera literaria y, con ello, se presentan como autónomas las prácticas literarias. Esta autonomía es la que da sentido a las prácticas literarias y permitc discriminar lo que es literario de lo que no es; el resultado es la mitificación de estas prácticas, junto con la noción de sujeto creador, la noción de crítico como exegeta, ctc. La base de esta legitimidad cs el aparato crítico capaz de enunciar leyes y sanciones sobre cstc campo. De acuerdo con Dubois (quien a su vez cita como fuentes a Sartre y a Barthes), la esfera de las prácticas literarias alcanza su autonomía en el siglo XIX, que es el siglo en el que la literatura obtiene una nueva estructuración, cuando se convicrte en asunto de hombres de le tras independientes y especializados, que crean sus códigos y sus reglas de trabajo y funcionamicnto. Dice Dubois que Sartre vio

por una parte, que la esfera literaria se plegaba sobre sí misma a favor de una ruptura en el orden simbólico y que, por otra parte, el escritor moderno participaba de una nueva clerecía, escribiendo para sus pares, es decir, principalmente para todos aque- 
llos que, como él y a títulos variados, se ocupaban de la cosa literaria (Dubois 1978: 22).

Ahora bien, si institución es todo lugar donde se opera la reproducción de las relaciones de dominación, podemos considerar a las instituciones como aparatos de poder; por lo tanto, si los discursos son producidos siempre por las instituciones, necesariamente estarán presentes en ellos ciertas relaciones de dominación: el discurso es el lugar privilegiado de ejercicio del poder porque es a través del discurso como se constituyen los sujetos y, a su vez, es en el sujeto donde el poder se ejerce en toda su profundidad. En las sociedades existen múltiples relaciones de poder que "atraviesan, caracterizan, constituyen el cuerpo social; y estas relaciones de poder no pueden disociarse, ni establecerse, ni funcionar sin una producción, una acumulación, una circulación, un funcionamiento del discurso" (Foucault 1978: 130-140). El orden del poder está en todo lugar; el poder no es sólo una fuerza que reprime, sino que debe considerarse como una red productiva que cruza todo el cuerpo social, que produce cosas, induce placer, forma saber, genera discursos; entre cada punto del edificio social, en la familia, en la escuela,

pasan relaciones de poder que no son la proyección pura y simple del gran poder del soberano sobre los individuos; son más bien el suelo movedizo y concreto sobre el que ese poder se incardina, las condiciones de posibilidad de su funcionamiento (Ibid: 157).

Esta concepción del poder es, por tanto, un poco distinta a la usual: en primer lugar, Foucault no localiza el poder en el aparato de Estado, es decir, en el conjunto de instituciones y aparatos que garantizan la sujeción de los ciudadanos a un Estado; tampoco es el sistema de dominación que ejerce un grupo o una clase social sobre otra: no es ni una institución, ni una estructura, ni una potencia de la que algunos están dotados, sino que el poder es "el nombre que se presta a una situación estratégica compleja en una sociedad dada" (Foucault 1977: 113). Por poder se entiende: 
-la multiplicidad de relaciones de fuerzal inmanentes y propias del dominio en que se ejercen, y que son constitutivas de su organización;

-el juego que, por medio de Iuchas, las transforma, refuerza e invierte;

- los apoyos que esas relaciones encuentran unas en otras, - las contradicciones que las aíslan;

-las estrategias que las hacen efectivas, cuya cristalización institucional toma forma en los aparatos estatales, en la formulación de las leyes, y en las hegcmonías sociales (Ibid: 112-113). El poder, de acuerdo con lo anterior, se produce en cada instante y en todos los puntos: cstá en todas partes porque viene de todas partes. El poder no es algo que se posea, sino algo que se ejerce: no es privilegio de un individuo o de una clase, sino que es el "cfecto que manifiesta y a veces acompaña la posición de aquellos que son dominados"' (Foucault 1976: 33). En resumen, el poder no se aplica a quicnes no lo tienen, sino que pasa por ellos, se apoya en ellos.

El poder cstá, pues, presente en los más finos mecanismos del intercambio social; como el espacio social es plural, dice Barthes, el poder es simétricamente perpetuo en el tiempo histórico. Para él, la razón de la persistencia y ubicuidad del poder es que éste es "cl parásito de un organismo transocial, ligado a toda la historia del hombre, y no solamente a su historia política. Este objeto en el que se inscribe el poder desde la eternidad humana es el lenguaje" (1980:3). Por lo tanto, los discursos, incluyendo allí los literarios, como manifestaciones de la capacidad de lenguaje son siempre portadores de relaciones de poder.

Sin embargo, las rclaciones de poder nunca son unívocas, sino que definen innumerables puntos de enfrentamiento, focos de inestabilidad que comportan conflictos, Iuchas, inversión de relaciones de fucrza. Por ello, todo discurso es espacio de lucha, pues donde hay poder hay resistencia: las relaciones de poder sólo pueden existir en función de una multiplicidad de puntos de resistencia. Barthes sostiene que nada escapa al poder, que dentro del lenguaje se cstá siempre sometido por lo que sólo se escapa al poder estando afuera del lenguaje; sin embargo, dice, como el lenguaje no tiene 
exteriores, "sólo nos queda hacer trampas con la lengua, hacerlc trampas a la lengua" y esto puede hacerse solamente en la literatura, en "ese señuelo magnífico que permite oír a la lengua fuera del poder". Esta conecepión romántica de la literatura comporta una visión monolítica del poder que no coincide con la desarrollada en párrafos anteriores: para Barthes toda literatura se opondría, por ser litcratura, al orden del discurso, sin tomar en cuenta lo institucional; de acuerdo con el punto de vista que aquí se sostiene, el discurso literario, en cuanto producido, difundido y recibido dentro de las normas de una institución, en tanto forma parte de una formación discursiva, reproduce también las rclaciones de poder.

Los discursos en general no están, en conclusión, ni totalmentc sometidos ni totalmente opuestos al poder; se trata más bien de

un juego complejo e inestable donde el discurso puede, a la vez, ser instrumento y efecto del poder, pero tạmbién obstáculo, tope, punto de resistencia y de partida para una estrategia opuesta. El discurso transporta y produce poder; lo refuerza, pero también lo mina, lo expone, lo torna frágil y permite detenerlo (Foucault 1977: 123).

Los discursos son, entonces, elementos o bloques tácticos en el campo de las relaciones de fuerzas; los discursos literarios no cscapan a esta posibilidad. Vcamos con más detalle este tipo de discurso.

Desde el punto de vista de la poética de Jakobson, hay dos factores que distinguen un discurso literario de los discursos no literarios, ellos son el autotelismo y el principio del paralelismo. Sabemos ahora que éstos: son factores nccesarios. pero no suficientes para ser literarios, pues hace falta el criterio de la función; es decir, además de poseer las características señaladas, deben desempeñar además la función de literatura en la sociedad. Pero dejemos de lado ahora ese problema.

Por otra parte, podemos hablar, además del orden que se establece por los tres grupos de procedimientos ya mencionados, de otro tipo de orden, que tienc que ver más bien con los 
contenidos manifiestos de los textos: es el que podríamos scñalar como la finalidad del discurso, para qué sirve y para quién. De acuerdo con Jakobson, los discursos litcrarios no ticnen una finalidad exterior a ellos, es decir, son autotélicos, tienen su fin en sí mismos; pero hay discursos producidos con un fin específico que posteriormente ingresan al corpus de textos que la literatura acoge como suyos: cartas, crónicas, etc., son ejemplos bien conocidos de este fenómeno. Lo contrario también puede encontrarse: discursos producidos con una intención literaria, que circularon y se leyeron como literatura - como algunos poemas de Bécquer o de Manuel Acuña- en la actualidad han dejado de funcionar y cuando se reproducen sirven para un fin determinado.

El principio del paralelismo, que establece que los discursos literarios poseen un mayor índice de estructuración, una mayor elaboración, una cantidad de trabajo incorporado en ellos más alta que la que se encuentra en discursos no literarios, tampoco es marca específica de literatura; en cambio, sí puede decirse que los textos reconocidos como literarios poseen en términos generales una mayor densidad en su aspecto material que los que no pertenecen a la literatura.

En resumen, ni el autotelismo ni la operación del principio del paralelismo hacen que un determinado discurso funcione como literario; sin embargo, todo texto literario cuenta con esas dos características. La base es, pues, el funcionamiento social de lo literario. Podríamos plantear, por lo tanto, que gracias a estas dos características, los discursos literarios son por excelencia los puntos de resistencia al poder; su mayor densidad -su más alta estructuración- los hace menos permeables a las relaciones de dominación, los hace opacos al poder. El texto literario -los textos artísticos en generaltienen un potencial subversivo, pues ponen en duda manifiestamente las nociones de origen, de creador, de legitimidad de la posesión de la fuerza, porque son las muestras palpables de que los procesos sociales no son continuos ni están. exentos de contradicciones. Por esta razón se requiere aplicar sobre ellos los procedimientos de control mencionados, esos poderosos mecanismos, pues es necesario conformarlos al. 
ordentel discurso, limitar su productividady su posibilidad de producir más de un significado.

Comoyy se dijo antes, todo discurso es también, simultáneamente, un no discurso; cada discurso es un espacio.de confrontación que se resuelve: a favor del discurso por medio de la puesta en acción de los prócedimientos de control. En los textos literarios, sin embargo -y esto quisiera ser la hipótesis que se propone en este trabajo-1a resolución en favor del orden no es tan automátical los textos literarios constituyen los lugares en los que la lucha entre discurso y no discurso es más encarnizada. Si los textos literarios son máquinas productoras de significaciones es porque no están sometidos totalmente, porque no se ha logrado convertirlos en discursos lineales, con una direccionalidad de lectura determinada: a pesar de los intentos pornormalizar a Cuesta y Artaud, a pesar de la prohibición de Bataille o de la descalificación de Revueltas; seguimos considerando que sus obras realizan las funciones de lo literario, pues siguen colmando necesidacies que sólo la literatura puede satisfacer. Paz dice que Becerra "trabaja una poesía quic empieza a deslizarse hacia temas a donde llegan; como ecos nostálgicos, las sensaciones más diferenciadas, las llamás de deseos que aún perduran en su conciencia"; no obstante, la poesía de Becerra sc resiste a sér solamente eso, y por ello seguimos leyéndola y considerándóla como parte de lo que nuestra cultura reconoce como literatura.

Desde el punto de vista adoptado en este escrito, puede hablarse de discurso literario para designar el conjunto de textos que funcionan como literatura; con ello se acentúa el hecho de que estos textos conforman ũno de los tipos de discursos presentes en nuestra sociedad; sin embargo, con esta designación también se borra el hecho de que en estos textos el conflicto discurso/no discurso, sujeción/no sujeción al orden, no está resuelto definitivamente. El uso indiscriminado de la expresión "discurso literario" presupone una toma de posición con respecto a lo literario.

CÉSAR GONZÁLEZZ.

Seminario de Poética.

Instituto de Investigaciones Filológicas IINAM 
ROLAND BARTHES. "Lección inaugural", Sábado 126, 1980.

José CARLos Becerra. El otoño recorre las islas, México: Era, 1973.

MICHEL B ERNARD. "Las condiciones del grupo de acción", en Análisis institucional y socioanálisis, México: Nueva Imagen, 1976.

J ACQUES D UBOIS. L'institution de la littérature, Bruselas: Labor, 1978.

GERARD D UROZOI. Artaud. L'aliénation et la folie, París: Larousse, 1972.

PIERRE F. EVRARD. "Las manos puras", en Análisis institucional y socioanálisis, ob. cit.

MICHEL F OUCAULT. El orden del discurso, Barcelona: Tusquets, 1973.

La arqueología del saber, México: Siglo XXI, 1976.

Vigilar y castigar, México: Siglo XXI, 1976.

. Historia de la sexualidad. 1. La voluntad de saber, México: Siglo XXI, 1977.

Microfisica dei joder, Barcclona: La piqueta, 1978.

J ENS I HWE. "On the validation of text-grammars in the 'study of literature' ", en Petöfi y Rieser (eds), Studies in Text Grammar, Dordrecht: Reidel, 1973.

RENE LOURAU. El análisis institucional, Buenos Aires: Amorrortu. MALDIDIER. Normand y Robin. "Discours et idéologie: quelques bases pour une recherche", Langue Française 15, 1972.

O CTAVIO P AZ. Poesía en movimiento, México: Siglo XXI, 1966. KARLHEINZ S TIERLE. "Identité du discours et transgressión lyrique", Poétique 32, 1977.

TZVETAN TODOROV". "La notion de littérature", en Langue, discours, societé. Pour Emile Benveniste, París: Seuil, 1975. "The Evolution of Poetics", Poétics 8 (6), 1979.

ELISEO VERON. "Sémiosis de l'idéologique et du pouvoir", Communications 28, 1978. 